\title{
Subthreshold channels at the edges of nanoscale triple-gate silicon transistors
}

\author{
H. Sellier, ${ }^{a)}$ G. P. Lansbergen, J. Caro, and S. Rogge \\ Kavli Institute of Nanoscience, Delft University of Technology, Lorentzweg 1, 2628 CJ Delft, \\ The Netherlands \\ N. Collaert, I. Ferain, M. Jurczak, and S. Biesemans \\ InterUniversity Microelectronics Center (IMEC), Kapeldreef 75, 3001 Leuven, Belgium
}

(Received 24 February 2006; accepted 13 January 2007; published online 12 February 2007)

\begin{abstract}
The authors investigate the subthreshold behavior of triple-gate silicon field-effect transistors by low-temperature transport experiments. These three-dimensional nanoscale devices consist of a lithographically defined silicon nanowire surrounded by a gate with an active region as small as a few tens of nanometers down to $50 \times 60 \times 35 \mathrm{~nm}^{3}$. Conductance versus gate voltage shows Coulomb blockade oscillations with a large charging energy due to the formation of a small potential well below the gate. According to dependencies on device geometry and thermionic current analyses, the authors conclude that subthreshold channels, a few nanometers wide, appear at the nanowire edges, hence providing an experimental evidence for the corner effect. (C) 2007 American
\end{abstract} Institute of Physics. [DOI: 10.1063/1.2476343]

Nonplanar field-effect transistors called FinFETs (Ref. 1) are currently being developed to solve the problematic issues encountered with the standard planar geometry when the channel length is reduced to a sub-100 nm size. Their triplegate geometry is expected to have a more efficient gate action and to solve the leakage problem through the body of the transistor, one of the dramatic short channel effects. ${ }^{2}$ However, their truly three-dimensional (3D) structure makes doping-and thus also potential-profiles very difficult to simulate and to understand using the current knowledge on device technology. Transport studies at low temperature, where the thermally activated transport is suppressed, can bring insight to these questions by measuring local gate action. For this reason we experimentally investigate the potential profile by conductance measurements and observe the formation of a subthreshold channel at the edge of the silicon nanowire. This corner effect has been proposed ${ }^{3,4}$ as an additional contribution to the subthreshold current in these 3D triple-gate structures, where the edges of the nanowire experience stronger gate action due to the geometric enhancement of the field. However, besides extensive simulation work ${ }^{3,4}$-keeping in mind the difficulties with these 3D structures-very little experimental work has been published until now on this effect.

The FinFETs discussed here consist of a narrow singlecrystalline silicon wire with two large contact pads etched in a $p$-type silicon on insulator layer doped with $10^{18} \mathrm{~cm}^{-3}$ boron atoms. This silicon wire is covered with a $t_{\mathrm{ox}}=1.4 \mathrm{~nm}$ thick thermal oxide and a second narrow polycrystalline silicon wire crossing the first one is fabricated to form a gate that surrounds the wire on three faces [Fig. 1(a)]. The entire surface is then implanted with $10^{19} \mathrm{~cm}^{-3}$ arsenic atoms to form $n$-type degenerate source, drain, and gate. During this implantation the wire located below the gate is protected and remains $p$ type. In the investigated device series the height of the fin wire is $H=60 \mathrm{~nm}$, while the width ranges from

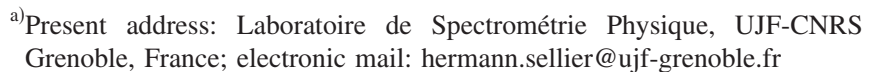

$W=35 \mathrm{~nm}$ to $1 \mu \mathrm{m}$ and the gate length ranges from $L=50 \mathrm{~nm}$ to $1 \mu \mathrm{m}$. The relatively high $p$-type doping of the channel wire is chosen to ensure a depletion length shorter than half the channel length in order to have a fully developed potential barrier in this $n-p-n$ structure and so to keep the conductance threshold at a large enough positive gate voltage. The characteristics at room temperature of these nanoscale FinFETs look therefore similar to those of their larger planar counterparts [Fig. 1(b) at $300 \mathrm{~K}$ ].

For subthreshold voltages, a barrier exists between the source and drain electron reservoirs and the transport is thermally activated at high enough temperature, as shown in Fig. 2(b). For very short devices, the conductance is simply given by the thermionic emission above the barrier according to the formula

$$
G=S A^{*} T\left(e / k_{B}\right) \exp \left(-E_{b} / k_{B} T\right),
$$

where the effective Richardson constant $A^{*}$ for $\mathrm{Si}$ is $2.1 \times 120 \mathrm{~A} \mathrm{~cm}^{-2} \mathrm{~K}^{-2}$. ${ }^{6}$ Several samples have been measured in this regime and their conductance has been fitted to obtain the barrier height $E_{b}$ and the cross section $S$ [Fig. 2(d)]. The two $385 \mathrm{~nm}$ wide samples have the same cross section
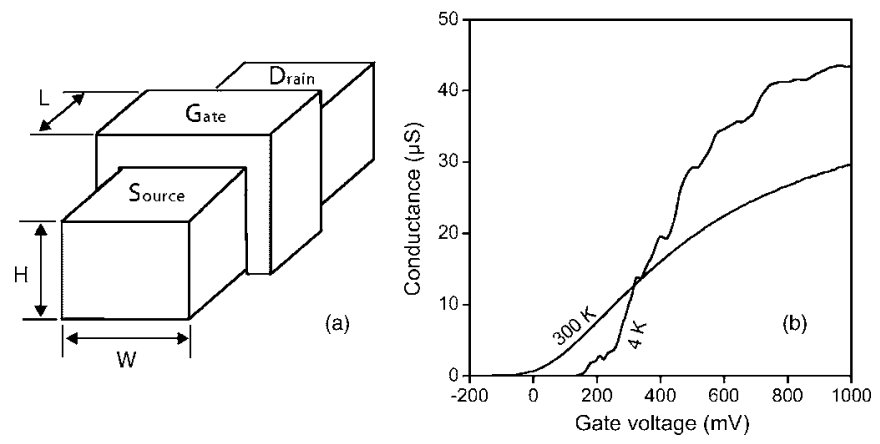

FIG. 1. (a) Schematic of the FinFET geometry where the gate surrounds the Si nanowire (the fin). (b) Low bias conductance vs gate voltage for a long and narrow silicon FinFET $(L=950 \mathrm{~nm}, W=35 \mathrm{~nm})$ at room and liquid helium temperatures. Reproducible conductance fluctuations appear due to quantum interferences. 

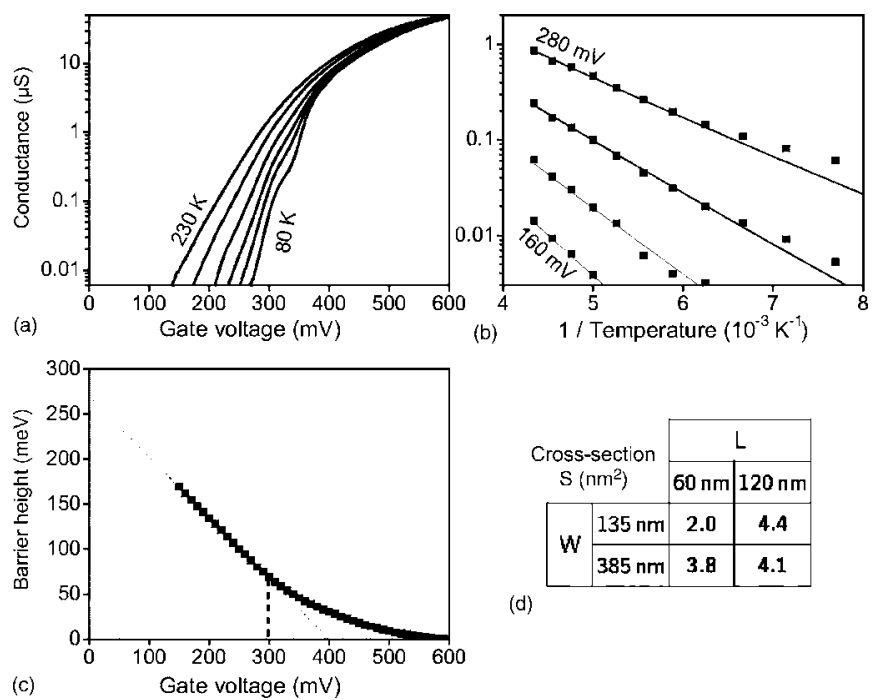

(c)

FIG. 2. (a) Conductance vs gate voltage (30 K steps) for a short and wide fin $(L=60 \mathrm{~nm}, W=385 \mathrm{~nm})$. (b) Conductance plotted vs the inverse of the temperature (40 $\mathrm{mV}$ steps) for the same sample. The conductance is thermally activated above $150 \mathrm{~K}$. (c) Barrier height vs gate voltage changing behavior at $300 \mathrm{mV}$ (same sample). (d) Measured cross section $S$ for the activated current of four samples with different lengths $L$ and widths $W$.

$S \approx 4 \mathrm{~nm}^{2}$ although their length differ by a factor of 2 . We can therefore conclude that transport is dominated by thermionic emission. The two $135 \mathrm{~nm}$ wide samples, however, have different $S$ values, but this cannot imply a diffusive transport since the longest sample has the largest conductance. Another result is that the cross section $S \approx 4 \mathrm{~nm}^{2}$ is much smaller than the channel width $W(135$ or $385 \mathrm{~nm})$ multiplied by the channel thickness (about $1 \mathrm{~nm}$ ). This result is consistent with the corner effect that produces a lower conduction band (stronger electric field) along the two edges of the wire, where the current will therefore flow preferentially

[Fig. 3(b)]. This interpretation is confirmed by the result obtained on a $385 \mathrm{~nm}$ wide sample with an undoped channel. Its larger cross section $S=24 \mathrm{~nm}^{2}$ (still much smaller than the width) is in agreement with the corner effect since the longer depletion length of the undoped silicon gives smoother potential variations and therefore wider channels along the edges.

The barrier height $E_{b}$ versus gate voltage is plotted in Fig. 2(c). The data extrapolated to zero gate voltage are consistent with a $220 \mathrm{meV}$ barrier height calculated for a $p$-type channel in contact with a $n^{++}$gate through a $1.4 \mathrm{~nm} \mathrm{SiO}{ }_{2}$ dielectric. ${ }^{6}$ The linear dependence of the barrier height shows a good channel/gate coupling ratio $\alpha=d E_{b} / e d V_{G}=0.68$ due to the triple-gate geometry with a thin gate oxide. At higher gate voltage (above $300 \mathrm{mV}$ ) the coupling ratio decreases and a finite barrier survives up to large voltages. Analysis of the low-temperature transport (see below) shows, however, that the gate action remains constant inside the channel where localized states are formed. The finite barrier is, in fact, two confining barriers located in the access regions (between channel and contacts), where the concentration of implanted arsenic atoms is reduced by the masking silicon nitride spacers placed next to the gate [Fig. 3(a).]

At low temperature the conductance develops fluctuations versus gate voltage [see Fig. 1(b)] with a pattern that reproduces after thermal cycling (at least for the main fea-
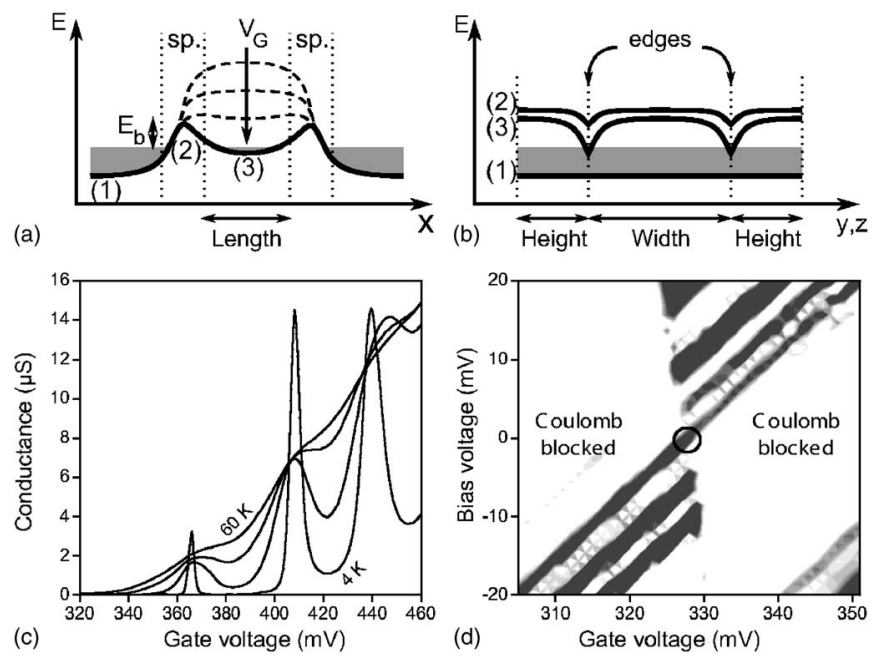

FIG. 3. (a) Conduction band edge profile with the highest barrier in the channel or in the access regions below the spacers (sp.) depending on gate voltage. (b) Band edge along the 3D gate oxide interface (1) in the contacts, (2) in the barriers, and (3) in the channel. The corner effect produces two channels with low barriers at the wire edges. (c) Conductance vs gate voltage for a short and wide channel $(L=60 \mathrm{~nm}, W=385 \mathrm{~nm})$ showing Coulomb blockade peaks up to high temperatures (20 K steps). (d) Stability diagram, i.e., conductance vs gate and bias voltages at $4.2 \mathrm{~K}$. The circle indicates a zero bias conductance peak, which develops into a triangular sector at finite bias.

tures). These fluctuations are caused by quantum interferences in the channel. For gate voltages close to the threshold, charge localization occurs, especially for short fins as can be seen in Fig. 3(c). When cooled down to $4.2 \mathrm{~K}$ the conductance pattern develops a series of peaks that we attribute to Coulomb blockade of electrons in the potential well created in the channel by the two tunnel barriers of the low-doped access regions. ${ }^{7,8}$ This interpretation is supported by the channel-length dependence of the peak spacing discussed later. An explanation in terms of a quantum well formed by an impurity can be ruled out. An impurity or defect could not accept many electrons, e.g., more than 20 for the $100 \mathrm{~nm}$ sample in Fig. 4(b), since they represent a single charge or empty state. These devices act therefore as quantum dots where the conduction electrons are spatially localized and are Coulomb blocked for the transport by a finite charging energy. ${ }^{9}$

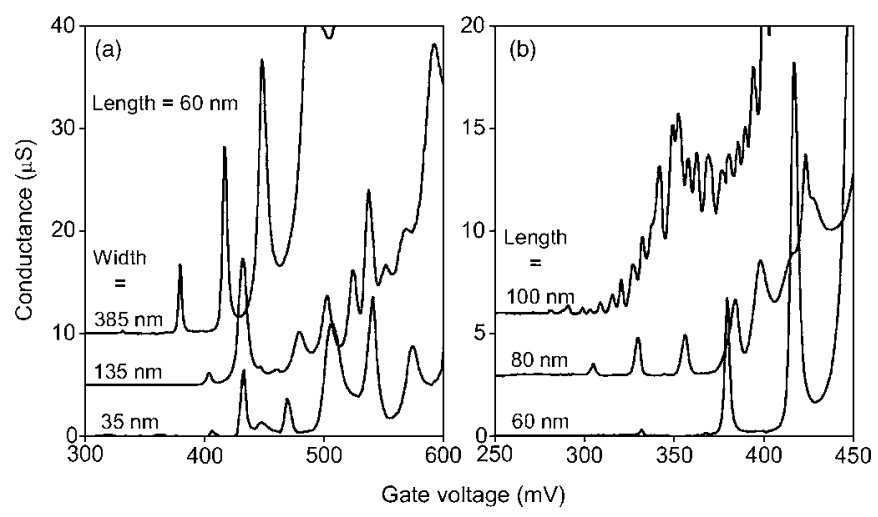

FIG. 4. Conductance vs gate voltage at $4.2 \mathrm{~K}$ for several devices. (a) Short fins $(L=60 \mathrm{~nm})$ of different widths $(W=35,135,385 \mathrm{~nm})$ have a similar peak spacing. (b) Devices with longer fins $(L=60,80,100 \mathrm{~nm})$ have a smaller peak spacing (the widths are different). The curves have been shifted for clarity. 
In the stability diagram of a quantum dot [see Fig. 3(d)], the slopes of a triangular conducting sector give the ratios of the capacitances $C_{G}, C_{S}$, and $C_{D}$ between the dot and, respectively, the gate, source, and drain electrodes. ${ }^{9}$ In this way we find the dot/gate coupling $\alpha=C_{G} /\left(C_{G}+C_{S}+C_{D}\right)=0.78(0.65)$ for the first (second) resonance. These values are close to the channel/gate coupling of 0.68 obtained independently in the same sample from the gate voltage dependence of the barrier height in the middle of the channel at higher temperatures. This result indicates that the gate coupling in the center of the device remains constant and supports the idea of a minimum in the conduction band, as sketched in Fig. 3(a).

The peak spacing $\Delta V_{G}$ is the change in gate voltage that increases by 1 the number of electrons in the dot located at the silicon/oxide interface. This quantity provides the dot/ gate capacitance $C_{G}=e / \Delta V_{G}$, and then the dot area $S=C_{G} / C_{\text {ox }}$ using the gate capacitance per unit area $C_{\mathrm{ox}}=\epsilon_{\mathrm{ox}} / t_{\mathrm{ox}}=0.025 \mathrm{~F} / \mathrm{m}^{2}$. The peak spacings for the same gate length $(L=60 \mathrm{~nm})$ but three different channel widths $(W=35,135$, and $385 \mathrm{~nm})$ can be compared in Fig. 4(a). Although the patterns are not very regular, an average peak spacing of about $30 \mathrm{mV}$ is obtained for all of them, indicating similar dot areas whereas the effective width is varied by more than a factor of 3 . On the opposite, the conductance patterns for three different lengths $(L=60,80$, and $100 \mathrm{~nm})$ shown in Fig. 4(b) have decreasing average peak spacings $\left(\Delta V_{G}=39,24\right.$, and $6 \mathrm{mV}$, respectively) and therefore increasing dot areas $\left(S=160,270\right.$, and $\left.1100 \mathrm{~nm}^{2}\right)$. However, these areas are not strictly proportional to the gate length, so that the actual width could be length dependent or the actual dot length could be smaller than the gate length for very short fins. If we assume that the dot length equals the gate length, we obtain 2.7, 3.4, and $11 \mathrm{~nm}$ for the dot width, i.e., a small fraction of the total Si/oxide interface width $W_{\text {eff }}=W+2 H$ $=150-500 \mathrm{~nm}$. The observation of similar dot widths of a few nanometers for different fin widths of hundreds of nanometers is consistent with the idea of a dot located at the edge of the fin and thus with the corner effect. ${ }^{3,4}$

In addition to a large charging energy $E_{c}=\alpha e \Delta V_{G}$, these dots also have a large quantum level spacing $\Delta E$, as can be deduced from the temperature dependence of the conductance peaks in Fig. 3(c). When the temperature is lowered below the level spacing, the tunneling process involves a single quantum level at a time and the peak height starts to increase above the high temperature value. ${ }^{10}$ The crossover from the classical to the quantum regime of Coulomb blockade being around $15 \mathrm{~K}$, we estimate the level spacing to be about $1.3 \mathrm{meV}$. If we use the gate length $L=60 \mathrm{~nm}$ in the expression $\Delta E=3 \pi^{2} \hbar^{2} / 2 m^{*} L^{2}$ for the energy separation between the first and second states of a one-dimensional system, we find a level spacing $\Delta E=1.6 \mathrm{meV}$ similar to the experimental estimation. This result supports the idea of a long dot extending over the whole gate length (assumed above to extract the dot width from the dot/gate capacitance).

In conclusion, both the activated current amplitude, the Coulomb blockade peak spacing, and the quantum level spacing reveal that the current flows in narrow channels a few nanometers wide. They appear along the edges of the FinFET due to an enhanced band bending called corner effect. In order to get a homogeneous current distribution with a lower subthreshold current and a larger on/off current ratio, this effect should be reduced. Better devices would have rounder corners on the scale of the depletion length and a lower doping concentration in the channel.

${ }^{1}$ D. Hisamoto, W.-C. Lee, J. Kedzierski, H. Takeuchi, K. Asano, C. Kuo, E. Anderson, T.-J. King, J. Bokor, and C. Hu, IEEE Trans. Electron Devices 47, 2320 (2000).

${ }^{2}$ M. C. Lemme, T. Mollenhauer, W. Henschel, T. Wahlbrink, M. Baus, O. Winkler, R. Granzner, F. Schwierz, B. Spangenberg, and H. Kurz, SolidState Electron. 48, 529 (2004).

${ }^{3}$ B. S. Doyle, S. Datta, M. Doczy, S. Hareland, B. Jin, J. Kavalieros, T. Linton, A. Murthy, R. Rios, and R. Chau, IEEE Electron Device Lett. 24, 263 (2003).

${ }^{4}$ J. G. Fossum, J.-W. Yang, and V. P. Trivedi, IEEE Electron Device Lett. 24, 745 (2003).

${ }^{5}$ W. Xiong, J. W. Park, and J. P. Colinge, IEEE Electron Device Lett. 25, 541 (2004).

${ }^{6}$ S. Sze, Physics of Semiconductor Devices, 2nd ed. (Wiley, New York, 1981), p. 257.

${ }^{7}$ F. Boeuf, X. Jehl, M. Sanquer, and T. Skotnicki, IEEE Trans. Nanotechnol. 2, 144 (2003)

${ }^{8}$ X. Jehl, M. Sanquer, G. Bertrand, G. Guegan, S. Deleonibus, and D. Fraboulet, IEEE Trans. Nanotechnol. 2, 308 (2003).

${ }^{9}$ L. P. Kouwenhoven, C. M. Marcus, P. L. McEuen, S. Tarucha, R. M. Westervelt, and N. S. Wingreen, Proceedings of the NATO Advanced Study Institutes (Kluwer, Dordrecht, 1997), Vol. 345, p. 105.

${ }^{10}$ C. W. J. Beenakker, Phys. Rev. B 44, 1646 (1991). 